\title{
Celiac Disease Diagnosis in Misdiagnosed Children
}

\author{
ANTONIO PICARELLI, LUIGI SABBATELLA, MARCO DI TOLA, FABIOLA GABRIELLI, ROSITA \\ GRECO, TERESA DI CELLO, ANTONIO MASTRACCHIO, AND MARIA CRISTINA ANANIA
}

\author{
Department of Clinical Sciences [A.P., L.S., M.D.T., F.G., R.G., T.D.C., M.C.A.] and Department of \\ Experimental Medicine [A.M.], University of Rome "La Sapienza," Rome, Italy
}

\section{ABSTRACT}

\begin{abstract}
Antiendomysial antibodies (EMA) are today considered the most sensitive and specific serological marker of celiac disease (CD). The aim of the present study was to assess the occurrence of EMA of IgG isotype in EMA IgA negative children with clinical suspicion of malabsorption and their relationship with CD. Serum EMA IgG1 determination was performed on 30 EMA IgA negative children with clinical suspicion of $\mathrm{CD}$. Total serum IgA levels were further investigated. Sixty children with gastroenterological diseases other than $\mathrm{CD}$ were used as control disease patients and 63 healthy children were evaluated as the control group. Eighteen out of 30 children in the study showed EMA IgG1 positivity in sera and a villous height/crypt depth ratio $<3: 1$ as index of intestinal atrophy. It is noticeable that a selective IgA deficiency was present in only 9 of 18 EMA IgG1 positive children. In addition, clinical symptoms, EMA IgG1, and mucosal atrophy disappeared after $8-10$ mo on a gluten-free diet. Neither EMA IgA nor EMA IgG1 were detected in the
\end{abstract}

children in the control groups. The other 12 children in study group showed no histologic abnormalities and were EMA IgG1 negative. In this study, we reveal a group of EMA IgG1 CD children without IgA deficiency. The diagnosis was based on the presence of gluten-dependent typical serological and histologic features of CD. Our data suggest that EMA IgG1 determination could be a new tool in the diagnostic workup of $\mathrm{CD}$, useful in avoiding possible misdiagnosis. (Pediatr Res 48: 590-592, 2000)
CD, celiac disease
EGDS, esophagogastroduodenoscopy
EMA, antiendomysial antibodies
AGA, antigliadin antibodies
IgG1, immunoglobulins, G1 subclass
GFD, gluten-free diet

Abbreviations
$\mathrm{CD}$ is a lifelong gluten intolerance characterized by villous flattening with crypt hyperplasia of the small bowel mucosa and improvement or normalization of the mucosal architecture after a GFD (1). The presence of circulating EMA, highly sensitive and specific for CD (2-5), and their disappearance after a GFD confirms the diagnosis (6). Therefore, EMA IgA are today accepted as a reliable and noninvasive screening test for $\mathrm{CD}$. Moreover, a recent study reported the presence of EMA of IgG isotype only in celiac children with selective IgA deficiency (7). These authors reported a low prevalence of AGA in these celiac children, suggesting the need to use different serological tests to reveal $\mathrm{CD}$ in the presence of $\operatorname{Ig} \mathrm{A}$ deficiency.

The aim of our study was to evaluate the occurrence of EMA $\operatorname{IgG}$ and their relationship to $\mathrm{CD}$ in a group of EMA $\operatorname{IgA}$ negative children with total or subtotal intestinal atrophy.

Received January 27, 2000; accepted July 3, 2000.

Correspondence: Antonio Picarelli, M.D., Department of Clinical Sciences, University of Rome "La Sapienza," Viale del Policlinico 155, Rome 00161, Italy.

\section{MATERIALS AND METHODS}

Subjects. Thirty EMA IgA negative children (12 males and 18 females, mean age 10.6, range 2-16 y) with clinical suspicion of CD were enrolled in the study as the patient group.

Sixty children (27 males and 33 females, mean age 11.3, range 4-16 y) with gastroenterological diseases other than CD (17 lactose intolerance, 3 cow's milk intolerance, 15 esophagitis, and 25 infectious diarrhea) were selected as the disease control group.

Sixty-three healthy children ( 30 males and 33 females, mean age 10.5, range 3-16 y) consisting of 25 patients with irritable bowel syndrome and 38 first-degree relatives of children with $\mathrm{CD}$, were also enrolled in the study.

All the patients at the time of admission in the study were on a gluten-containing diet.

Informed consent was obtained from all subjects under study. The study was approved by the applicable institutional review board.

Detection of EMA. EMA were sought in sera diluted 1:5 by means of indirect immunofluorescence analysis on cryostat sections of monkey esophagus (Eurospital SpA, Trieste, Italy). 
FITC-conjugated anti-human IgG1 (Sigma Chemical Co., St. Louis, MO, U.S.A.) were used diluted 1:100. Positive EMA results were identified by reticulin-like staining of smoothmuscle bundles (Fig. 1).

Detection of $\boldsymbol{A G} \boldsymbol{A}$. AGA were measured in sera, diluted 1:100 for AGA IgA and 1:500 for AGA IgG, by an in-house ELISA (8). Alkaline-phosphatase-conjugated goat anti-human IgA and IgG (Dako, Copenhagen, Denmark), diluted 1:1000, were used. OD values were obtained by enzyme immunoassay (EIA) reader at $405 \mathrm{~nm}$.

AGA values were expressed as OD sample/OD pool normal serum +3 SD. The cut-off values, 0.9 for AGA IgA and 1.1 for AGA IgG, were identified by using a receiver operating characteristic analysis (8).

Detection of total IgA. Total IgA immunoglobulins were measured by a radial immune-diffusion method (Diffuplate, Bioscentifica, Buenos Aires, Argentina) and results were evaluated by referring to a standard curve. Normal values ranged between 90 and $220 \mathrm{mg} / \mathrm{dL}$ and, in accordance with the manufacturer's instructions, $90 \mathrm{mg} / \mathrm{dL}$ was used as a cut-off point to identify IgA deficiency.

Histologic analysis. Two biopsy specimens of duodenal mucosa were obtained from each child in study by EGDS, for diagnostic purposes. The villus height/crypt depth ratio was measured and values below 3:1 were considered pathognomonic of CD.

Follow-up. All of the EMA IgG1 positive children were kept on a GFD, and compliance with the diet was checked by a monthly serum EMA detection. When EMA IgG1 positivity and clinical signs and symptoms disappeared, in accordance with European Society for Pediatric Gastroenterology and Nutrition criteria (9), a second EGDS for histologic analysis was performed.

\section{RESULTS}

Eighteen of the 30 EMA IgA negative patients with clinical suspicion of CD showed total or subtotal intestinal mucosa atrophy with a villus height/crypt depth ratio below $3: 1$. No histologic abnormalities were found in the other 12 children in the patient group (Table 1).

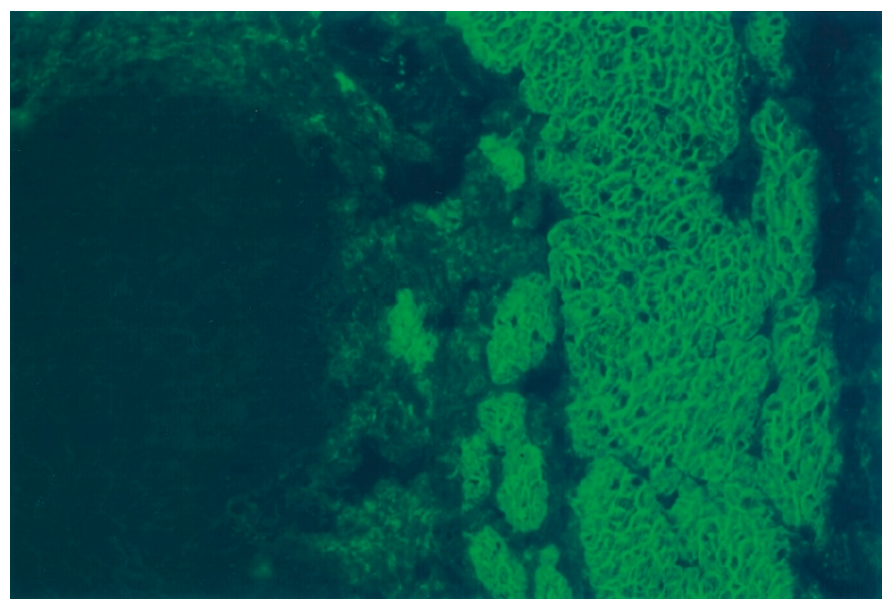

Figure 1. Serum EMA IgG1 positive. A feature of EMA is a honeycomb-like fluorescence pattern along muscolaris mucosae of monkey esophagus.
Table 1. Histological, serum EMA, and AGA positive results

\begin{tabular}{|c|c|c|c|c|c|}
\hline \multirow[b]{2}{*}{ Subjects } & \multirow{2}{*}{$\begin{array}{l}\text { Intestinal } \\
\text { atrophy* }\end{array}$} & \multicolumn{2}{|c|}{$\begin{array}{c}\text { EMA } \\
\text { positivity }\end{array}$} & \multicolumn{2}{|c|}{$\begin{array}{c}\text { AGA } \\
\text { positivity }\end{array}$} \\
\hline & & $\operatorname{Ig} \mathrm{A}$ & IgG1 & $\operatorname{IgA}$ & $\mathrm{IgG}$ \\
\hline \multicolumn{6}{|l|}{ Patients group $(n=30)$} \\
\hline CD patients $(n=18)$ & $18 / 18$ & $0 / 18$ & $18 / 18$ & $4 / 18$ & $6 / 18$ \\
\hline Negative patients $(n=12)$ & $0 / 12$ & $0 / 12$ & $0 / 12$ & $4 / 12$ & $5 / 12$ \\
\hline \multicolumn{6}{|l|}{ Control groups } \\
\hline Disease control group $(n=60)$ & - & $0 / 60$ & $0 / 60$ & - & - \\
\hline Healthy children $(n=63)$ & - & $0 / 63$ & $0 / 63$ & - & - \\
\hline
\end{tabular}

$*$ Villus height/crypt depth ratio $<3: 1$.

EMA of IgG1 isotype were found in all of the 18 EMA IgA negative children presenting with total or subtotal intestinal mucosa atrophy. On the other hand, no EMA, IgA, or IgG1 were detected in the 12 children with clinical suspicion of $\mathrm{CD}$ who did not show intestinal mucosa atrophy, in the 60 disease controls, or in the 63 healthy children (Table 1).

AGA were measured in all 18 EMA IgG1 positive children and in all 12 children with EMA IgA and who were EMA IgG1 negative. AGA of IgA class were positive in 4 of 18 children with intestinal mucosa atrophy and in 4 of 12 EMA negative children, whereas AGA of IgG class was positive in 6 of 18 children with intestinal mucosa atrophy and in 5 of 12 EMA negative children (Table 1).

Sensitivity and specificity of AGA IgA determination were $22.2 \%$ and $66.7 \%$, respectively, whereas for AGA IgG determination, sensitivity and specificity were $33.3 \%$ and $58.3 \%$, respectively.

Normal levels of total serum IgA were found in 9 of 18 EMA IgG1 positive children (50\%), therefore, 9 children were considered as having serum IgA deficiency.

In all 18 EMA IgG1 positive children studied, EMA IgG1 disappeared within 4-6 mo on a GFD, and a complete clinical remission was observed within 8-10 mo of gluten withdrawal. The second EGDS, performed when EMA IgG1 were not more detectable, showed in all these patients a complete recovery of the mucosal villi architecture after a GFD.

\section{DISCUSSION}

$\mathrm{CD}$, defined as a permanent intolerance of the small intestine to gluten, is characterized by gluten-dependent changes in villous morphology and/or signs of immunologic activation of the lamina propria with serum antiendomysial antibodies (10, 11). Although EMA are today considered the most specific serological marker of $\mathrm{CD}$, with a specificity approaching to $100 \%$, their sensitivity ranges between $74 \%$ and $100 \%(2-5)$. Nonetheless, although EMA IgG have been reported in the occurrence of selective IgA deficiency (7), EMA currently used in the diagnostic work-up of $\mathrm{CD}$ are of the IgA class. Therefore, data emerging from this report reveal a group of children with symptoms typical of $\mathrm{CD}$ and intestinal atrophy presenting EMA IgG1 in the absence of EMA IgA positive results. Anti-human IgG1 detection was selected in relation to the known distribution of $\operatorname{IgG}$ subclasses in humans $(12,13)$. Furthermore, unlike to the observations reported in the literature (7), our data showed that EMA IgG1 occurred also in the 
absence of IgA deficiency. Therefore, the presence of EMA $\mathrm{IgG1}$ is not always linked to impaired $\operatorname{IgA}$ production but is probably due to a different isotypic switch leading to the development of B-cell clones producing EMA IgG1 instead of IgA.

As previously reported in the literature (7), our study further showed that AGA had very low sensitivity and specificity in the population of EMA IgG1 positive children. This observation confirms that EMA IgG1 determination is necessary to reveal subjects already misdiagnosed using other serological tests. Furthermore, the absence of EMA IgG1 in the control groups determines a high value for the specificity of EMA IgG1 test in $\mathrm{CD}$.

It is noticeable that in all of these EMA IgG1 positive children, intestinal atrophy was found. Moreover, clinical symptoms, EMA IgG1 positivity, and mucosal atrophy disappeared within 8-10 mo of a correct GFD, showing their strict gluten dependency and therefore suggesting that EMA IgG1 patients have $\mathrm{CD}$.

$\mathrm{CD}$ has been likened to an iceberg because of the large number of people suffering from this condition who elude correct diagnosis (14). Our data identifies a group of EMA IgG1 CD patients, without IgA deficiency but with the same characteristics described for the typical EMA IgA positive CD patients (1), which could be representative of the submerged part of that iceberg. In fact, EMA IgG1 evaluation could increase the sensitivity of EMA testing, allowing the identification of CD patients who may be missed using EMA IgA test alone. Thus, we suggest the introduction of EMA IgG1 determination as new serological tool useful in the diagnostic work-up of CD.

\section{REFERENCES}

1. Maki M, Collin P 1997 Celiac disease. Lancet 349:1755-1759

2. Ferreira M, Davies SL, Butler M, Scott D, Clark M, Kumar P 1992 Endomysial antibody: is it the best screening test for celiac disease? Gut 33:1633-1637

3. Corrao G, Corazza GR, Andreani ML, Torchio P, Valentini RA, Galatola G, Quaglino D, Gasbarrini G, Di Orio F 1994 Serological screening of celiac disease: choosing the optimal procedure according to various prevalence values. Gut 35:771-775

4. Bottaro G, Volta U, Spina M, Rotolo N, Sciacca A, Musumeci S 1997 Antibody pattern in childhood celiac disease. J Pediatr Gastroenterol Nutr 24:559-562

5. Valdimarsson T, Frazen L, Grodizinsky E, Skogh T, Strom M 1996 Is small bowel biopsy necessary in adults with suspected celiac disease and IgA anti-endomysium antibodies? $100 \%$ positive predictive value for celiac disease in adults. Dig Dis Sci 41:83-87

6. Kumar V, Lerner A, Veleski JE, Beutner EH, Chorzelski TP, Rossi T 1989 Endomysial antibodies in the diagnosis of celiac disease and the effect of gluten on antibody titers. Immunol Invest 18:533-544

7. Korponay-Szabo IR, Kovacs JB, Czinner A, Goracz G, Vamos A, Szabo T 1999 High prevalence of silent celiac disease in preschool children screened with $\operatorname{IgA} / \operatorname{IgG}$ antiendomysium antibodies. J Pediatr Gastroeneterol Nutr 28:26-30

8. Picarelli A, Triglione P, Mariani P, Di Giovambattista F, Greco M, Gurnari M, Paoluzi P, Bonamico M, Ballati G 1996 Use of a threshold serum level of anti-gliadin antibodies improves diagnostic efficiency of the test in adult coeliac disease but is unreliable as a screening test. Ital J Gastroenterol 28:70-75

9. Walker-Smith J, Guandalini S, Schmitz J 1990 Report of Working Group of European Society for Pediatric Gastroenterology and Nutrition: revised criteria for diagnosis of coeliac disease. Arch Dis Child 65:909-911

10. Maiuri L, Picarelli A, Boirivant M, Coletta S, Mazzilli MC, De Vincenzi M, Londei M, Auricchio S 1996 Definition of the initial immunologic modifications upon in vitro gliadin challenge in the small intestine of celiac patients. Gastroenterology 110:13681378

11. Picarelli A, Maiuri L, Mazzilli MC, Coletta S, Ferrante P, Di Giovambattista F, Greco M, Torsoli A, Auricchio S 1996 Gluten-sensitive disease with mild enteropathy. Gastroenterology 111:608-616

12. Klouche M, Bradwell AR, Wilhelm D, Kirchner H 1995 Subclass typing of IgG paraproteins by immunofixation electrophoresis. Clin Chem 41:1475-1479

13. Lee FK, Nahmias AJ, Spira T, Keyserling H, Lowery S, Reimer C, Black C, Stoll B, Czerkinski C 1991 Enumeration of human peripheral blood lymphocytes secreting immunoglobulins of major classes and subclasses in healthy children and adults. J Clin Immunol 11:213-218

14. Catassi C, Ratsch IM, Fabiani E, Rossini M, Bordicchia F, Candela F, Coppa GV, Giorgi PL 1994 Celiac disease in the year 2000, exploring the iceberg. Lancet 343:200-203 